Check for updates

Cite this: RSC Adv., 2019, 9, 16366

Received 4th April 2019

Accepted 13th May 2019

DOI: $10.1039 / c 9 r a 02558 \mathrm{~h}$

rsc.li/rsc-advances

\section{New chair shaped supramolecular complexes- based aryl nicotinate derivative; mesomorphic properties and DFT molecular geometry}

\author{
H. A. Ahmed, (iD *ab M. Hagar (D) *ac and O. A. Alhaddad ${ }^{\text {d }}$
}

A new series of chair-shaped liquid crystalline complexes were formed through $1: 1$ intermolecular hydrogen bonding between 4-(4-(hexyloxyphenylimino)methyl)phenyl nicotinate and 4-alkoxybenzoic acids, with different alkoxy chains. The mesomorphic behaviour of these supramolecular hydrogenbonded complexes was investigated through differential scanning calorimetry and polarizing optical microscopy. The Fermi bands of the hydrogen bonding formation were confirmed through Fouriertransform infrared spectroscopy. Schlieren textures of the nematic phase $(N)$ were observed for all prepared complexes. These thermotropic supramolecular complexes were confirmed also using density functional theory calculations. It was found that as the akoxy chain length increased the predicted total energy of the $\mathrm{H}$-bonded complex also increased. The predicted data of the charge distribution explained the nematic mesophase which covered all $1: 1$ mixtures and it could be attributed to more end-to-end aggregations of the alkoxy chains with longer chain lengths. Moreover, formation of the $\mathrm{H}$-bonded complexes greatly affected polarizability with respect to the individual compounds. The polarizability increased three and one third times compared with that of the free acid and base, respectively.

\section{Introduction}

Molecular interactions resulting from hydrogen bonding liquid crystal systems ${ }^{\mathbf{1 - 6}}$ recently have received more attention in applicable research. These types of mesomorphic materials involve non-covalent interactions and have potential applications for functional molecular structures. Molecular geometry is one of the important factors affecting the properties of liquid crystalline materials $^{7-9}$ i.e., mesophase formation and stability. Supramolecular hydrogen bonding liquid crystals (SMHBLCs) are one of the most important inductions in chemical and biological processes for aggregation and association of molecules. SMHBLCs are mostly based on pyridyl and carboxylic derivatives, as proton acceptor and proton donor moieties, respectively. ${ }^{10-13}$ Schiff base compounds are widely used in many fields, such as catalytic reactions and photochemistry. ${ }^{\mathbf{1 4 , 1 5}}$ Many Schiff bases with a low molar mass have been synthesized and investigated extensively for applications with liquid crystals. ${ }^{16-19}$ Generally, stability of a mesophase depends on the polarity and/or polarizability of the central part of the molecule. High demands of new SMHBLC complexes for applications has

${ }^{a}$ College of Sciences, Chemistry Department, Taibah University, Yanbu, Saudi Arabia. E-mail: ahoda@sci.cu.edu.eg; mohamedhaggar@gmail.com

${ }^{b}$ Faculty of Science, Department of Chemistry, Cairo University, Cairo, Egypt

${ }^{c}$ Faculty of Science, Chemistry Department, Alexandria University, Alexandria, Egypt

${ }^{d}$ College of Sciences, Chemistry Department, Madina Monawara, Taibah University, Saudi Arabia led to the preparation and study of numerous mesogens, particularly thermotropic liquid crystals. ${ }^{20-23}$ Several mesogenic homologous series containing ester/Schiff base central linkages have been widely reported. ${ }^{\mathbf{1 6 , 1 8 , 1 9 , 2 4 - 2 6}}$ Most of the H-bonded mesogens studied are based on rod-like intermolecular $\mathrm{H}$ bonding ${ }^{20-23,27,28}$ and there is an increasing interest for forming angular SMHBLCs through intermolecular hydrogen-bond formation. ${ }^{29,30}$

Nowadays, designing materials according to computational prediction also has attracted high interest. ${ }^{24-26,31-37}$ To understand many optical properties requires simulated information about molecular geometries as well as energies of the molecular orbitals of liquid crystalline compounds. Recently, density functional theory (DFT) has become an effective method because of its excellent performance and consistent computational results.

Continuing our systematic work, the goal of the present study was to design new chair-form Schiff base/ester supramolecular materials with new mesomorphic and optical properties. A series of Schiff base/ester linkage derivatives I(A-E) were prepared through hydrogen-bonding formation between 4-(4(hexyloxyphenylimino)methyl)phenyl nicotinate (I) as proton acceptors and 4-alkoxybenzoic acids (A-E) as proton donors (see the structure below) and their thermal and mesomorphic properties were investigated. In addition, we determined the effects of the length of alkoxy chains of the acid moiety on mesophase stability of the supramolecular complexes. On the other hand, we planned to use the DFT calculations to predict 
the thermal parameters of the free compounds as well as their supramolecular complexes. Moreover, the relationship between the experimental and the theoretical predictions confirmed our results, especially, the mesophase type and stability of the formed complexes.

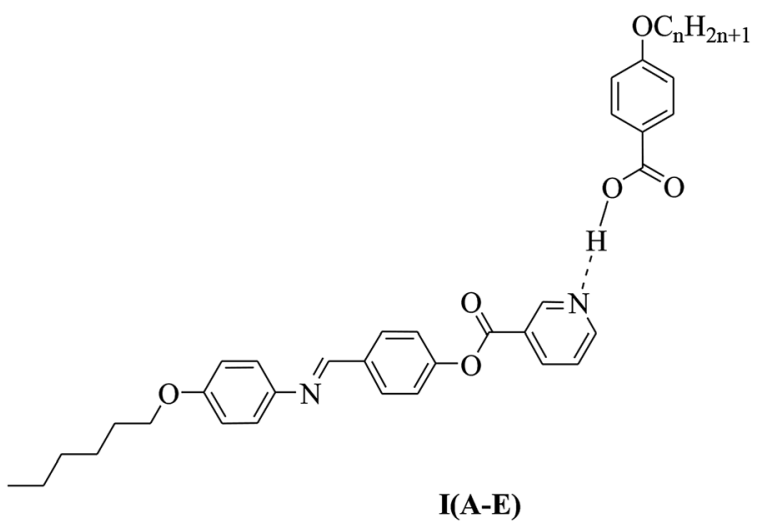

\section{Experimental}

\subsection{Materials}

4-Alkoxybenzoic acids were obtained from Merck (Germany) while nicotinic acid, $N, N^{\prime}$-dicyclohexylcarbodiimide (DCC), 4hydroxybenzaldehyde, 4-hexyloxyaniline and 4-dimethylaminopyridine (DMAP) were purchased from Aldrich (Wisconsin, USA) and used as received. All solvents used were pure grade and purchased from Aldrich (Wisconsin, USA).

A TA Instruments Co. Q20 Differential Scanning Calorimeter (DSC; USA) was used for calorimetric measurements. The DSC was calibrated using the melting temperature and enthalpy of indium and lead. The DSC investigation was carried out using small samples $(2-3 \mathrm{mg})$ placed in aluminum pans. All measurements were achieved with a heating rate of $10^{\circ} \mathrm{C} \mathrm{min}^{-1}$ in an inert atmosphere of nitrogen $\left(30 \mathrm{~mL} \mathrm{~min}^{-1}\right)$ and all transitions were recorded with a second heating scan from room temperature to $280{ }^{\circ} \mathrm{C}$.

Transition temperatures for the individual components and their $1: 1$ associated complexes were determined by DSC, and types of the mesophase were identified with a standard polarised optical microscope (POM, Wild, Germany) attached to a Mettler FP82HT hot stage. The temperature was measured by a thermocouple attached to the temperature controller. Measurements were made twice and the results have an accuracy in the transition temperature within $\pm 0.2{ }^{\circ} \mathrm{C}$.

\subsection{Preparation of pyridine-based derivative}

2.2.1. Synthesis of 4-((4-alkoxyphenylimino)methyl)phenol (A). A mixture of 4-hexyloaniline derivatives $(790 \mathrm{mg}, 4.1 \mathrm{mmol})$ and 4-hydroxybenzaldehyde $(0.5 \mathrm{~g}, 4.1 \mathrm{mmol})$ were dissolved in ethanol $(10 \mathrm{~mL})$. The solution was heated under reflux for two hours, and then allowed to cool to room temperature for complete precipitation. The obtained precipitate was filtered and washed with cold ethanol and recrystallized from hot ethanol.
2.2.2. Synthesis of 4-(4-(hexyloxyphenylimino)methyl) phenyl nicotinate (I). A mixture of 4-dimethylaminopyridine (DMAP) (trace amount) and $N, N^{\prime}$-dicyclohexylcarbodiimide (DCC, $0.02 \mathrm{~mol})$ was added to a solution of 0.01 mole 4-(4alkoxyphenylimino)methyl)phenol (I) and nicotinic acid (1.23 g, $0.01 \mathrm{~mol}$ ) in $25 \mathrm{~mL}$ dry methylene chloride. The reaction mixture was kept under stirring at room temperature for 72 hours after which the separated byproduct, $N, N$-dicyclohexylurea, was filtered. The filtrate was then evaporated to dryness. The obtained solid product was purified by recrystallization twice from ethanol (Scheme 1).

2.2.3. Preparation of $1: 1$ supramolecular complexes. Supramolecular complexes (I(A-E)) in $1: 1$ molar ratios of any two complementary components were prepared by melting appropriate amounts of each component, stirring to give an intimate blend and then, cooling with stirring to room temperature (Scheme 2).

\subsection{Characterization}

Purity of I was preliminarily checked with thin-layer chromatography using TLC-sheets coated with silica gel (E. Merck) and the spots were detected by a UV-lamp. Their molecular formulae were confirmed via elemental analyses, Fourier-transform infrared spectroscopy (FTIR, Nicolet is 10 Thermo scientific) and ${ }^{1} \mathrm{H}$-NMR spectroscopy (Varian EM 350L $300 \mathrm{MHz}$ spectrometer, Oxford, UK).

Formation of the supramolecular complexes (I(A-E)) were confirmed by DSC investigation as well as FTIR.

\subsection{Computational methods and calculations}

Theoretical calculations for the investigated compounds were carried out using Gaussian 09 software..$^{38}$ DFT/B3LYP methods using the 6-31G $(\mathrm{d}, \mathrm{p})$ basis set was selected for the calculations. Geometries were optimized by minimizing the energies with respect to all geometrical parameters without imposing any molecular symmetry constraints. The structures of the optimized geometries were drawn with Gauss View..$^{39}$ Moreover, the calculated IR frequencies were carried out using the same level of theory. The frequency calculations showed that all structures were stationary points in the geometry optimization method with no imaginary frequency.

\section{Results and discussion}

\subsection{Experimental and theoretical conformation of SMHBLCs formation}

Infrared spectra measurements were performed for the individual components i.e., 4-alkoxybenzoic acids (A-E), 4-(4-(hexyloxyphenylimino)methyl)phenyl nicotinate (I) as well as to their supramolecular complexes (I(A-E)). The experimental data from the FTIR spectra of $\mathbf{I}$ and $\mathbf{B}$ and their complex IB are given in Fig. 1 as representative examples. The results revealed that the alkoxy chain length of the benzoic acids (A-E) had no significant effect on the wavenumber of the spectral band of $\mathrm{C}=\mathrm{O}$ and were found at wavenumber $1680 \mathrm{~cm}^{-1}$. The dimeric form of the benzoic acid derivatives (A-E) under intermolecular 


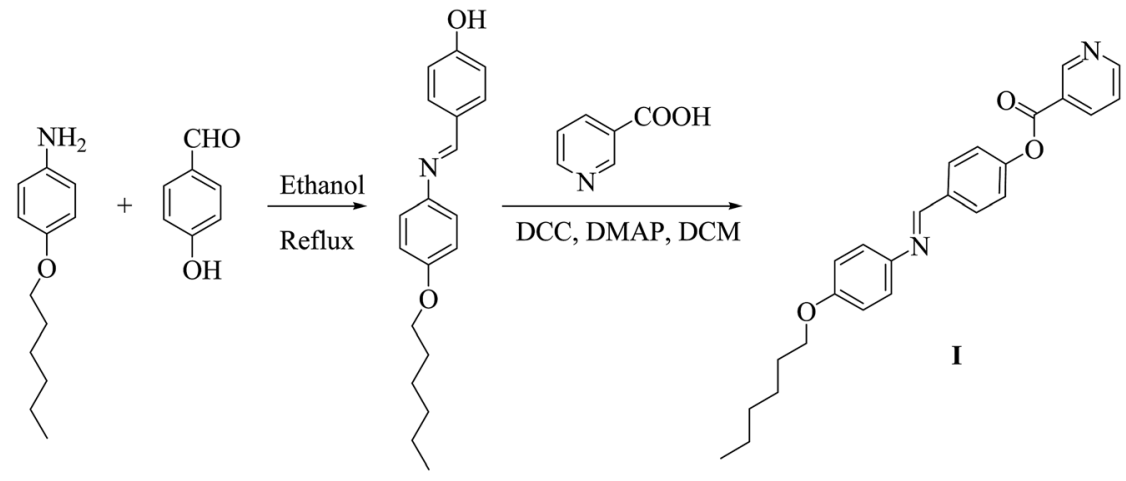

A

Scheme 1 Preparation of 4-(4-(hexyloxyphenylimino) methyl)phenyl nicotinate (I).

hydrogen bonding between the carboxylic groups was replaced by another intermolecular hydrogen bonding between the pyridyl moiety of base I and the carboxylic acid. This new intermolecular hydrogen bonding was confirmed by experimental and theoretical data from IR spectra.

The $\mathrm{C}=\mathrm{O}$ stretching vibration is an important piece of evidence for identifying intermolecular $\mathrm{H}$-bonding, where participation of the hydrogen atom from the $\mathrm{O}-\mathrm{H}$ of the carboxylic group increases the strength of the $\mathrm{C}=\mathrm{O}$ group and consequently, its stretching is shifted to a higher wavenumber $\left(\nu=1689 \mathrm{~cm}^{-1}\right)$. This shift could be attributed to the fingerprint of a heterocomplex formation between the nicotinate and alkoxy acids. Moreover, the formation of the complex increases the strength of the $\mathrm{C}=\mathrm{O}$ of the ester linkage of the base and its wavenumber increased by $19 \mathrm{~cm}^{-1}, \nu=1729$ to $1748 \mathrm{~cm}^{-1}$. Additionally, the stretching vibration from the $\mathrm{C}=\mathrm{N}$ of the pyridyl group was slightly decreased by only $4 \mathrm{~cm}^{-1}$ under $\mathrm{H}$ bonding. On the other hand, it has been reported ${ }^{40-46}$ that three Fermi resonance vibration bands with A-, B-, and C-types of the hydrogen bonded $\mathrm{OH}$ groups are another important evidence for formation of the H-bonded complex. A vibrational peak which appeared and overlapped with that of the $\mathrm{C}-\mathrm{H}$ bond vibrations could be attributed to the A-type Fermi band, while another at $2356 \mathrm{~cm}^{-1}$ corresponds to a B-type due to the $\mathrm{O}-\mathrm{H}$ inplane bending vibration as well as its fundamental stretch. However, a peak at $1911 \mathrm{~cm}^{-1}$ could be attributed to an interaction between the overtone of the torsional effect and the fundamental stretching vibration of the $\mathrm{OH}$, C-type. The predicted values of the wavenumbers of the characteristic groups are tabulated in Table 1. The calculated values in Table 1 were scaled according to the reported method. ${ }^{47}$ The results of the DFT calculation of the predicted wavenumbers are consistent with the experimental data. The lower values of the theoretical wavenumbers could be attributed to absence of an intermolecular interaction between molecules in the gas phase that were used in the theoretical calculations.

\subsection{Mesomorphic and optical properties of individual components and their complexes}

Mesomorphic behaviors of the present phenyl nicotinate (I) and their $1: 1$ supramolecular complexes (I(A-E)) were investigated by DSC and POM. Texture observations by POM were verified by DSC measurements and mesophase types were identified for individual and all prepared supramolecular complexes.

3.2.1. 4-(4-(Hexyloxyphenylimino)methyl)phenyl nicotinate, I. Temperature and enthalpy of transitions for the prepared nicotinate derivatives (I) are given in Table 2. The data revealed that the phase behavior of $\mathbf{I}$ is monomorphic exhibiting an enantiotropic nematic (N) phase. DSC thermograms for I upon second heating/cooling cycles are depicted in Fig. 2. It is clear that a broad range of nematic phase stability is exhibited, where it melts at $130.7^{\circ} \mathrm{C}$ and changes to the isotropic liquid at

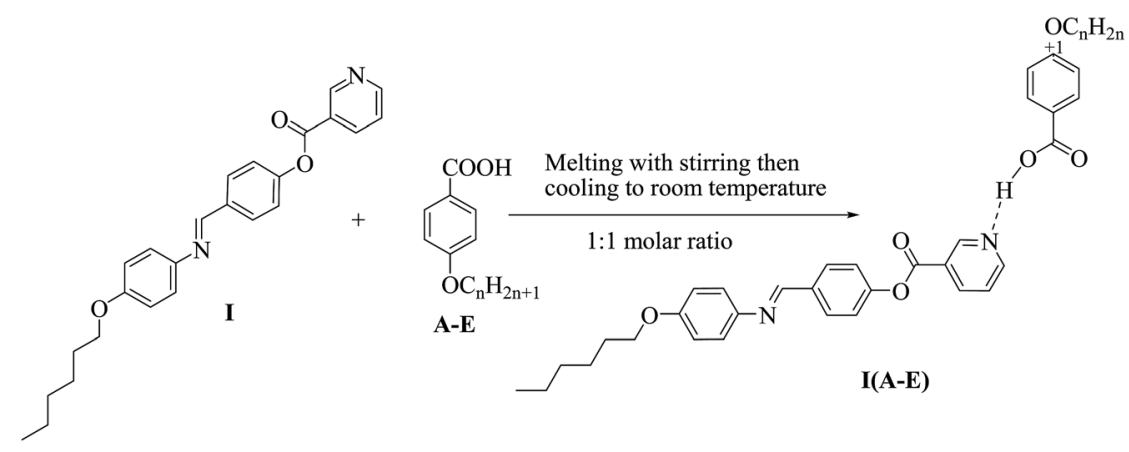

Scheme 2 Preparation of $1: 1$ supramolecular hydrogen-bonded complexes (I(A-E)). 

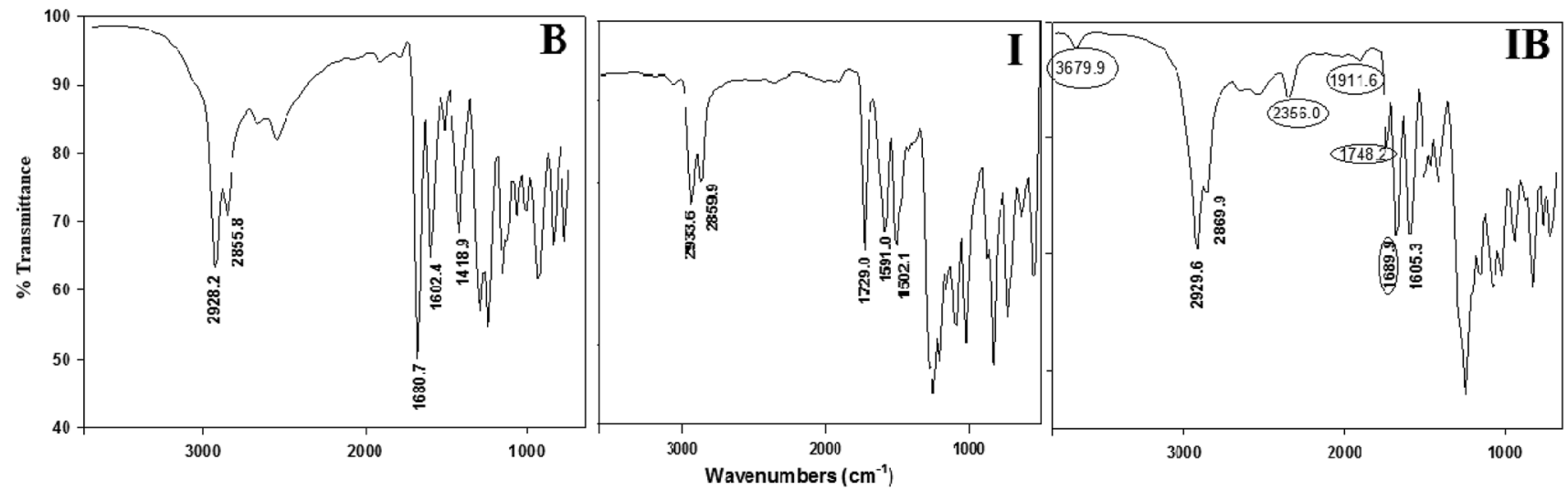

Fig. 1 FT-IR spectra of B, I and IB.

$183.6{ }^{\circ} \mathrm{C}$. On the other hand, the nematic phase was the only mesophase observed by cooling the isotropic melts.

3.2.2. (1:1) Molar supramolecular hydrogen-bonding complexes, I(A-E). While phenyl nicotinate $\mathbf{I}$ is purely nematogenic and the 4-n-alkoxybenzoic acids are mesomorphic with two mesophases, smectic $\mathrm{C}$ and nematic, the type of the phase depends on the terminal chain length. ${ }^{48}$ Therefore, it was interesting to investigate the mesomeric behavior of their angular supramolecular complexes (I(A-E)) to study the effect of the akoxy chain length of the acid moiety on the mesophase behavior of complexes prepared and predict the geometrical structures of the supramolecular complexes to deduce the most probable conformation shape. For the 1:1 molar supramolecular complexes $\mathbf{I} / \mathbf{A}-\mathbf{E}$, transition temperatures and their corresponding enthalpy of transitions also were collected in Table 2. Phase transition temperatures are graphically represented in Fig. 3. As seen from Table 2 and Fig. 3, as the alkoxy chain length $(n)$ increased the melting temperatures of the prepared compounds were irregularly changed and the mesophase stability decreased. Moreover, all complexes that exhibited nematogenic mesophases appeared enantiotropically. Supramolecular hydrogen-bonding complexes (IA, IB, IC, ID and IE), exhibited mesophase thermal stabilities of 37.4, 36.4, 39.8, 32.8, and $22.4{ }^{\circ} \mathrm{C}$ upon heating, respectively. Fig. 3 also shows that for a given pyridine-based complement, the IC mixture exhibits the lowest melting temperature in addition to a wide nematic phase range which was constructed via intermolecular hydrogen bonding. It is obvious that the $\mathrm{N}$ thermal transition decreases gradually with increasing alkoxy chain length $(n)$ in agreement with previously reported data by Gary et al. ${ }^{49}$ and Imire et al. ${ }^{50}$ The display of $\mathrm{N}$ phases and their stability is ascribed to protracted molecular lengths which increase the polarity to
Table 2 Phase transition temperatures $\left({ }^{\circ} \mathrm{C}\right)$, enthalpy of transitions $(\mathrm{kJ}$ $\left.\mathrm{mol}^{-1}\right)$, and transition entropy for the supramolecular complexes I(AE) ${ }^{a}$

\begin{tabular}{lrllll}
\hline System & $T_{\mathrm{Cr}-\mathrm{N}}$ & $\Delta H_{\mathrm{Cr}-\mathrm{N}}$ & $T_{\mathrm{N}-\mathrm{I}}$ & $\Delta H_{\mathrm{N}-\mathrm{I}}$ & $\Delta S / R_{\mathrm{N}-\mathrm{I}}$ \\
\hline I & 130.7 & 35.52 & 184.6 & 1.91 & 1.24 \\
IA & 92.2 & 40.28 & 129.6 & 2.09 & 1.94 \\
IB & 91.7 & 52.48 & 128.1 & 3.41 & 3.21 \\
IC & 86.9 & 77.68 & 126.7 & 3.99 & 3.79 \\
ID & 92.5 & 90.23 & 125.3 & 4.04 & 3.88 \\
IE & 100.9 & 84.07 & 123.3 & 2.43 & 2.37
\end{tabular}

${ }^{a}$ Abbreviations: $T_{\mathrm{Cr}-\mathrm{N}}=$ crystal to nematic phase transition; $T_{\mathrm{N}-\mathrm{I}}=$ nematic to isotropic liquid transition. $\Delta H_{\mathrm{Cr}-\mathrm{N}}=$ crystal to nematic phase transition; $\Delta H_{\mathrm{N}-\mathrm{I}}=$ nematic to isotropic liquid transition. $\Delta S /$ $R_{\mathrm{N}-\mathrm{I}}=$ nematic to isotropic liquid transition.

polarizability ratio, permanent dipole moment, dipole-dipole interactions and intermolecular dispersion forces. ${ }^{24}$ As the lengths of alkoxybenzoic acids terminals increase the central core rigidity of the supramolecular H-bonding will decrease, consequently, the linearity of the complexes decreases to some extent due to the greater number of configurations of the chains and then a strong terminal interaction predominates to give a nematic phase. Representative textures of the mesophases under a polarizing optical microscopic are shown in Fig. 4.

Entropies of the nematic-isotropic transitions $\left(\Delta S / R_{\mathrm{N}-\mathrm{I}}\right)$ were calculated for all the supramolecular mixtures. The results are tabulated in Table 2, and represented graphically as a function of the acid alkoxy-chain length $(n)$ in Fig. 5. From the results of Table 2, the entropy change $\left(\Delta S / R_{\mathrm{N}-\mathrm{I}}\right.$, Fig. 5$)$ increases with the increments of the alkoxy chain $n$ up to $n=12$ carbons $\left(\Delta S / R_{\mathrm{N}-\mathrm{I}}\right.$ $=3.88)$ then jumps to decrease at $n=16\left(\Delta S / R_{\mathrm{N}-\mathrm{I}}=2.37\right)$. That

Table 1 The theoretical (experimental) wavenumbers $\left(\mathrm{cm}^{-1}\right)$ of the characteristic groups of I, B and IB

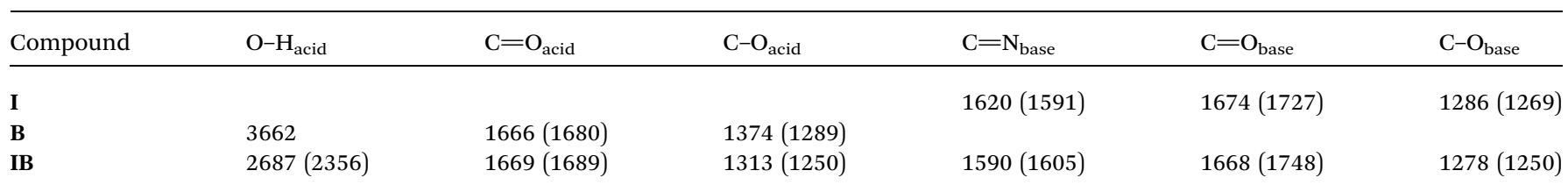




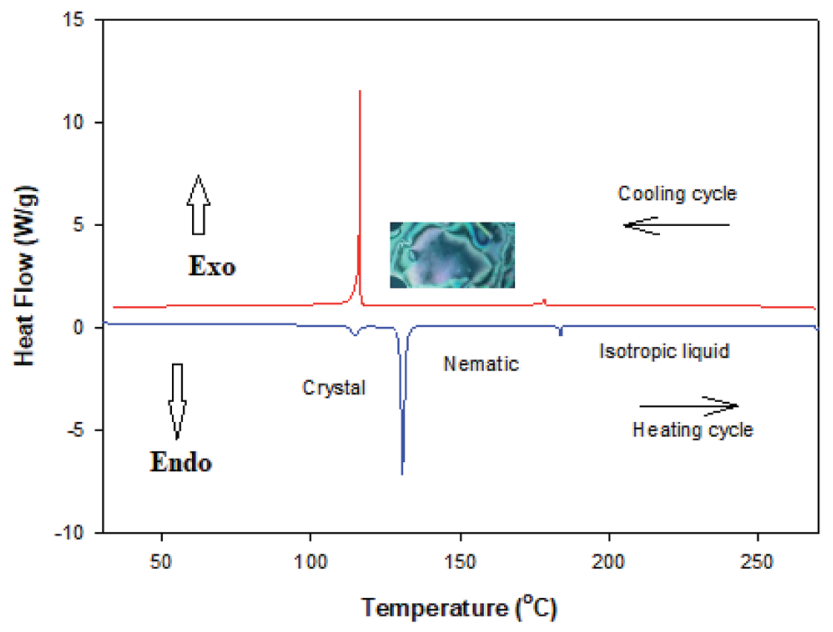

Fig. 2 DSC thermogram for I upon second heating/cooling cycles with heating rate $10^{\circ} \mathrm{C} \mathrm{min}^{-1}$.

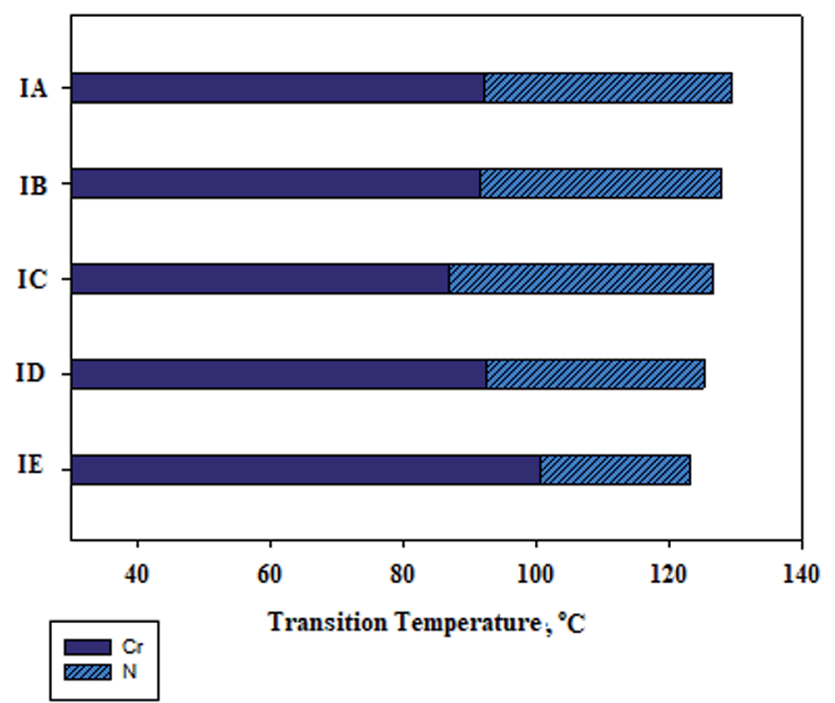

Fig. 3 DSC graphical transitions of supramolecular complexes series I(A-E) on heating cycles.

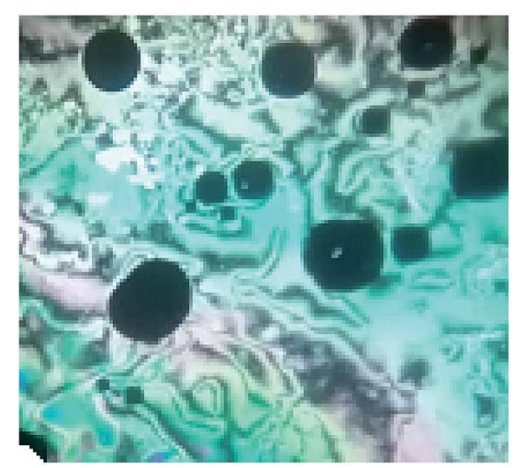

Fig. 4 Nematic mesophase texture under a polarized optical microscope for supramolecular complex IB at $126^{\circ} \mathrm{C}$ upon heating.

irregular relation is due to an increase of the end-to-end aggregation of molecules with an increase in acid alkoxychain length as a result of the formation of the less ordered $\mathrm{N}$

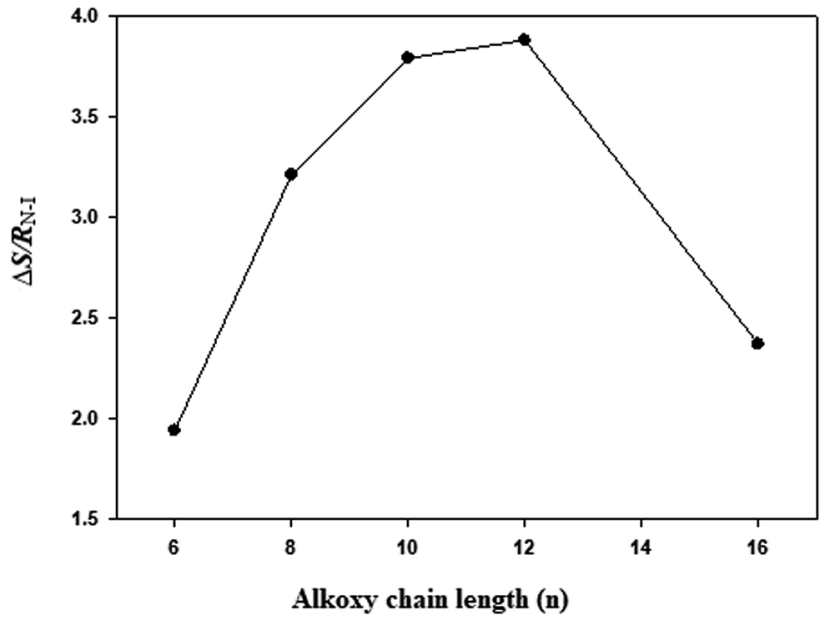

Fig. 5 Correlation between entropy changes of nematic transition $\left(\Delta S / R_{\mathrm{N}-1}\right)$ and alkoxy chain length $(n)$ of the acid component.

phase. That arrangement of molecules led to a decrease of the apparent dipole moment value of their observed nematic mesophase. Moreover, the entropy changes during phase transitions are relatively high which is attributed to a high conjugative interaction with the mesogenic portion of the molecules.

\subsection{DFT calculations}

3.3.1. Geometries. Theoretical calculations were performed in the gas phase for base $\mathbf{I}$ and acid $\mathbf{B}$ as well as prepared $\mathrm{H}^{-}$ bonded supramolecular complexes (IA, IB, IC, ID, IE) using method DFT/B3LYP at basis set 6-31G (d,p). Absence of the imaginary frequency for all optimized structures is approval of their stability.

As shown from Fig. 6, although acid $\mathbf{B}$ and base $\mathbf{I}$ have linear shapes, the geometry of the prepared complexes is not linear. All of them take a non-linear shape which could be considered a "chair form" (Fig. 7). This geometry could illustrate the covering of the nematic phases for all prepared H-bended complexes for all chain lengths. It is well known ${ }^{48}$ that 4 alkoxy acids with a shorter chain length $(n=6)$ exhibit a nematic phase while the longest alkoxy chain length $(n=16)$ is smectogenic and exhibits only the smectic phase. This data could be explained in terms of an increment of the lateral interaction as the chain length increases. The linear shape of the acids permits the maximum side-side interaction. Similarly, due to the short chain length of the base, the strong end to end aggregation of the chains could be the overriding effect of the nematic mesophase of the base. However, the chair forms of the prepared complexes do not permit the strong lateral interaction, thus leaving end to end aggregation of the chains to be the pronounced interaction. This projection could be a good explanation for the nematic mesophases observed for all alkoxy chain lengths of the $\mathrm{H}$-bonded complexes.

3.3.2. Thermal parameters. Values of thermal parameters for all H-bonded complexes were calculated with the same method using the same basis set and are tabulated in Table 3 . Fig. 8 shows the relationship between nematic stability of the 

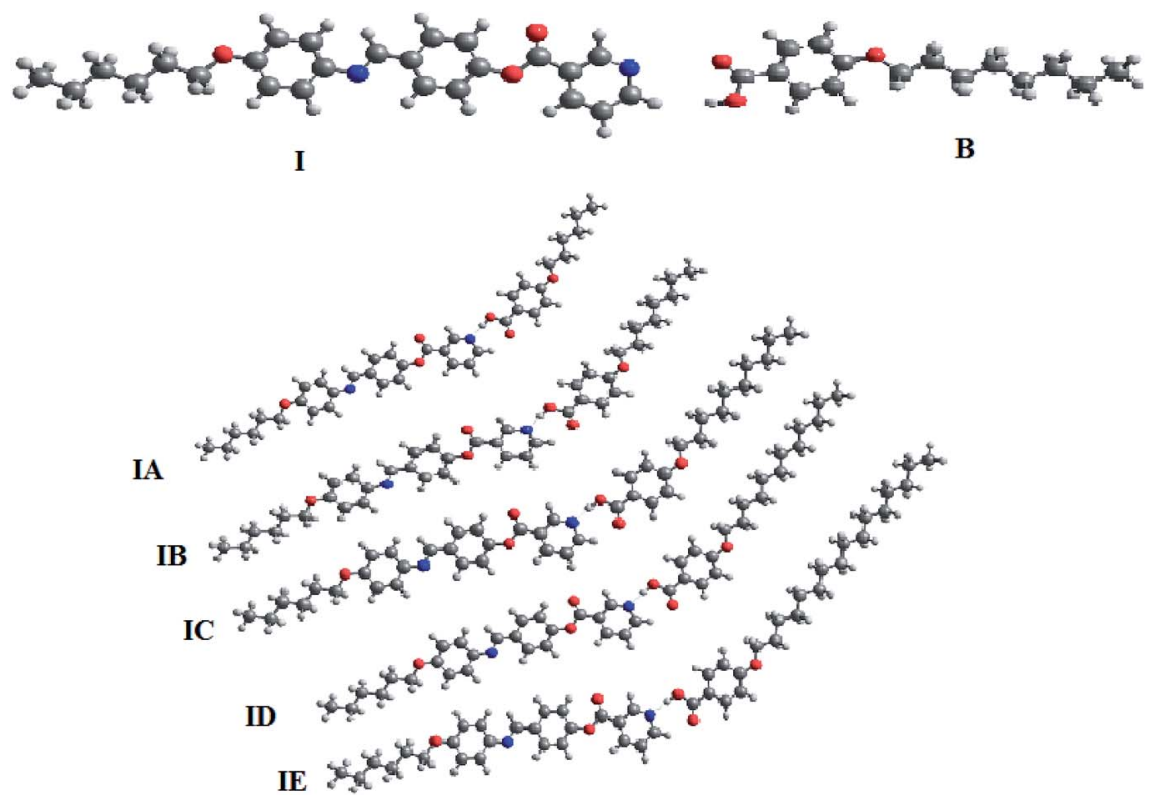

Fig. 6 Calculated geometrical structures of I, B, IA, IB, IC, ID and IE.

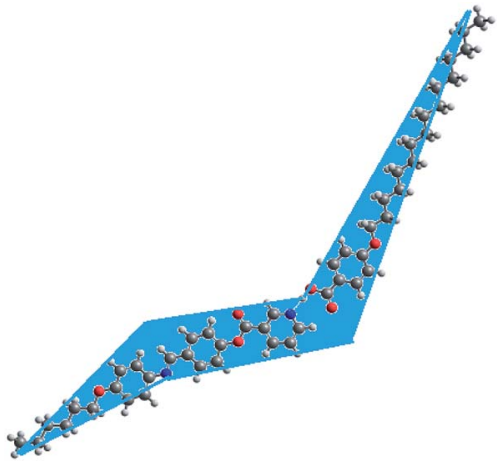

Fig. 7 Chair form projection of compound IE.

prepared complexes with the estimated energy of the H-bonded complexes. It may be concluded that as the alkoxy chain length increases, the predicted total energy of the H-bonded complex also increases while the mesophase stability decreases. This result could be attributed to stronger aggregation of the alkoxy chains with longer lengths. The stronger terminal aggregation

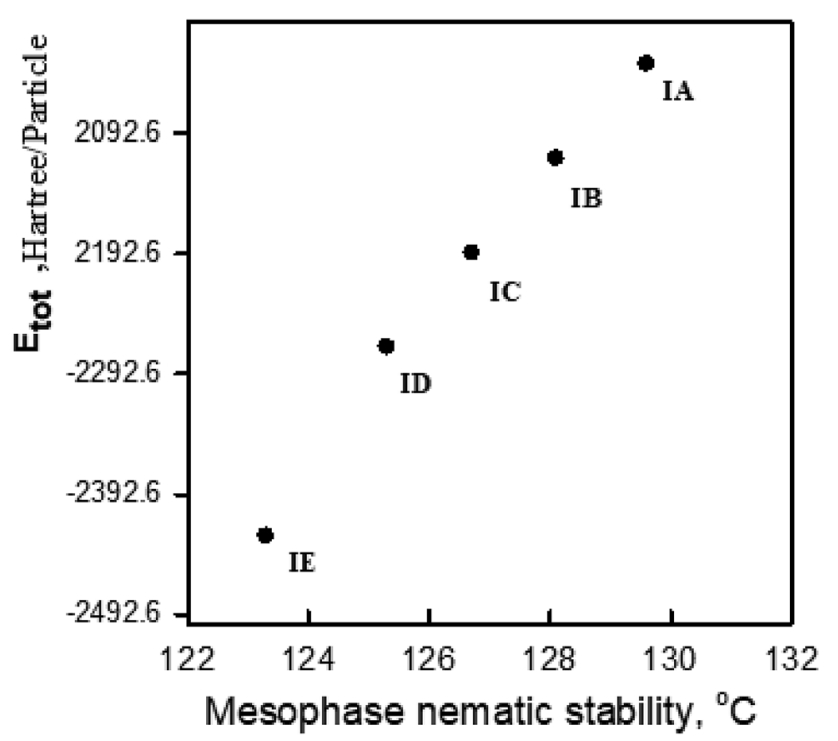

Fig. 8 Relationship between the sum of the electronic $\&$ thermal energies and the nematic stability of supramolecular complexes, I(AE).

Table 3 Thermal parameters (hartree per particle) of IA, IB, IC, ID and IE ${ }^{a}$

\begin{tabular}{|c|c|c|c|c|c|}
\hline Parameter & IA & IB & IC & ID & IE \\
\hline$E_{\text {corr }}$ & 0.752451 & 0.809630 & 0.866701 & 0.923748 & 1.038004 \\
\hline ZPVE & -2034.600163 & -2113.167181 & -2191.734306 & -2270.301442 & -2427.435932 \\
\hline$E_{\text {tot }}$ & -2034.554664 & -2113.119003 & -2191.683399 & -2270.247803 & -2427.376020 \\
\hline$H$ & -2034.553720 & -2113.118058 & -2191.682454 & -2270.246859 & -2427.375075 \\
\hline$G$ & -2034.691970 & -2113.262393 & -2191.833640 & -2270.404840 & -2427.549529 \\
\hline$\Delta H$ & 392.82136 & 314.25702 & 235.69262 & 157.12822 & 0 \\
\hline
\end{tabular}

${ }^{a}$ ZPVE: sum of electronic and zero-point energies; $E_{\text {tot }}$ : sum of electronic and thermal energies; $H$ : sum of electronic and thermal enthalpies; $G$ : sum of electronic and thermal free energies. 

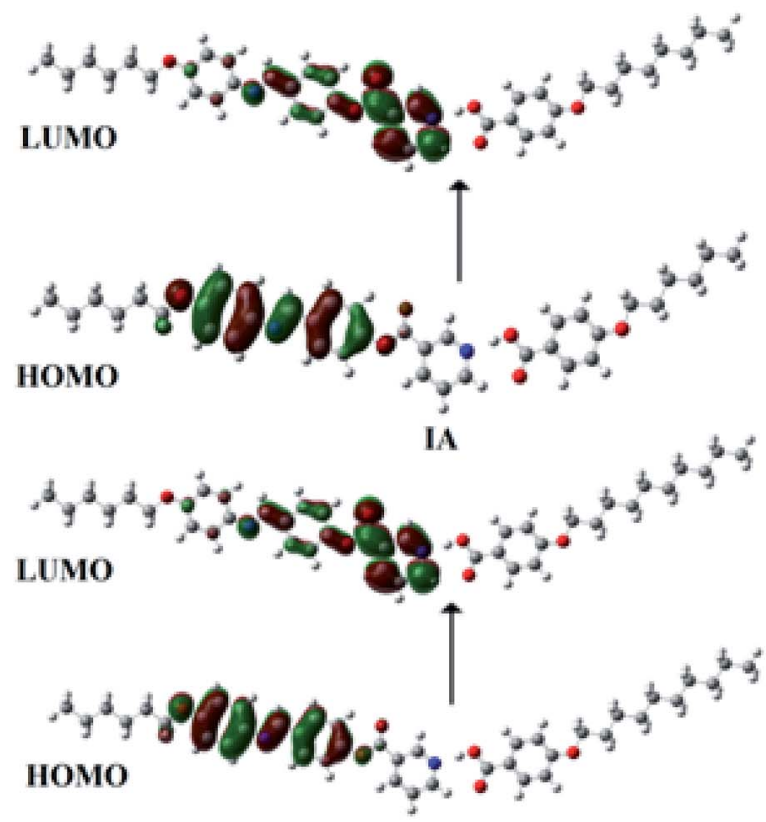

IC

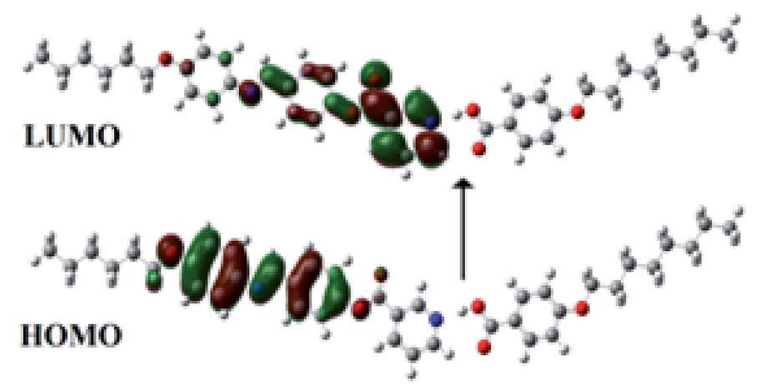

IB

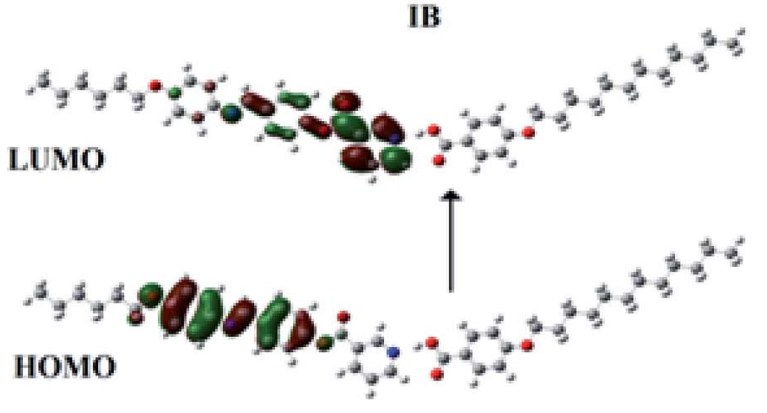

ID

Fig. 9 Estimated plots for Frontier molecular orbitals of IA, IB, IC and ID.

increases the calculated total thermodynamic energy and decreases the nematic stability.

3.3.3. Frontier molecular orbitals and polarizability. Polarizability and energy difference of Frontier molecular orbitals, HOMO (highest occupied) and LUMO (lowest unoccupied), are important characteristics for liquid crystalline compounds that may be used in many applications such as telecommunications, signal processing and optical interconnections. ${ }^{51,52}$ Moreover, nonlinear optical (NLO) liquid crystalline compounds need high polarizability for good applications. ${ }^{53}$ As shown from Fig. 9, electron densities of the HOMO and LUMO are mainly localized on the base part and there is no sharing of the alkoxy acid in these molecular orbitals. This data could illustrate independence of the Frontier molecular orbital with the alkoxy chain length. The calculated energy differences and the ground state isodensity surface plots for FMOs of all the $\mathrm{H}$-bonded complexes and the free compounds are tabulated in Table 4. Similarly, there is no effect of the length of an alkoxy chain on the total dipole moment. On

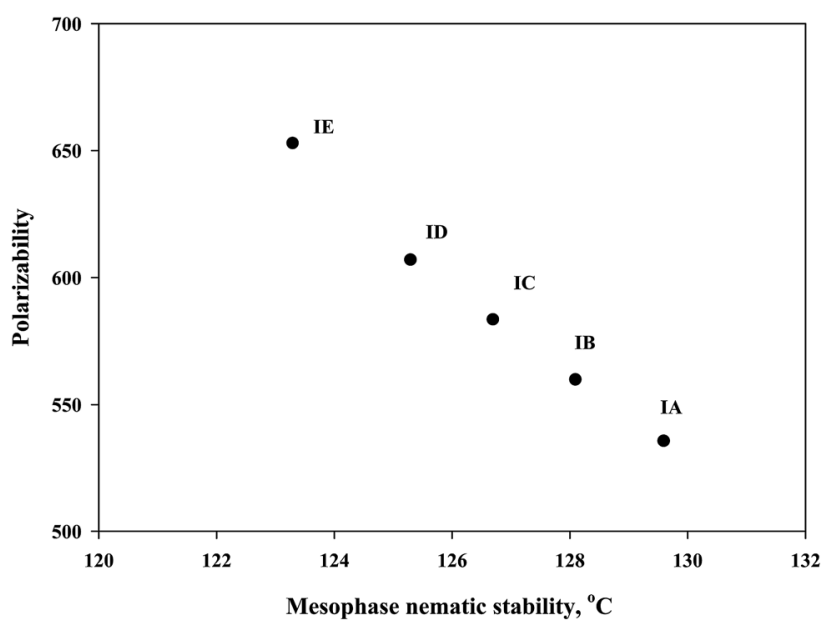

Fig. 10 Correlation between polarizability and nematic stability of supramolecular complexes, I(A-E) with different chain lengths of alkoxy acids.

Table 4 FMO energies a.u., polarizability, $\alpha$, and dipole moments $\mu$ (Debye) of I, B, IA, IB, IC, ID and IE

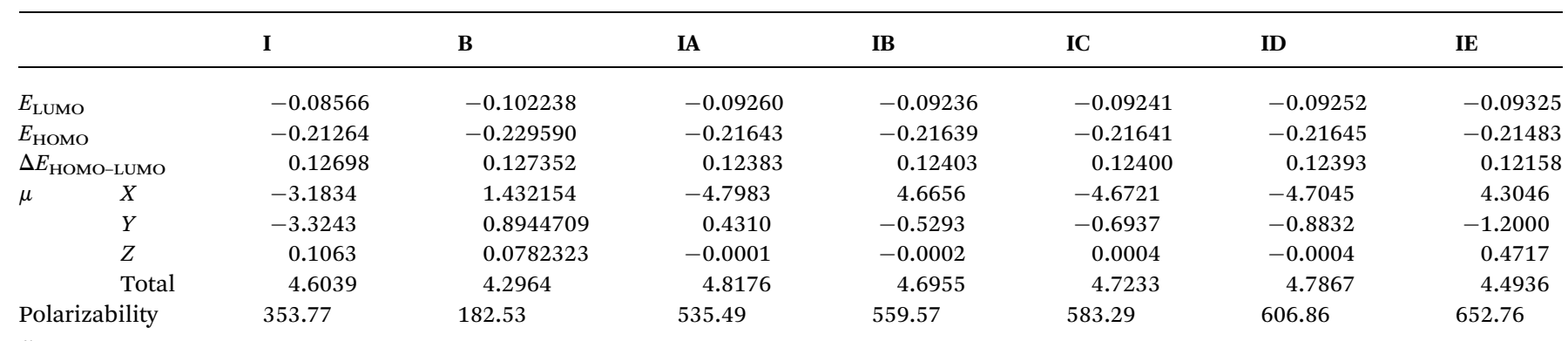




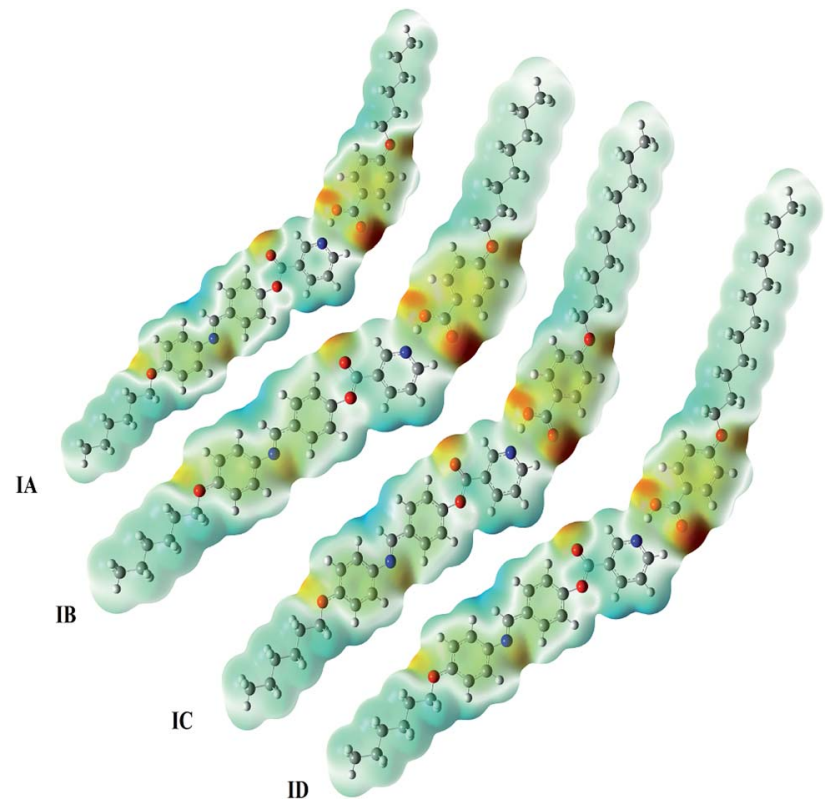

Fig. 11 Molecular electrostatic potentials (MEP) of IA, IB, IC and ID.

the other hand, formation of the H-bonded complexes highly affects polarizability with respect to the free compounds. Polarizability increases three and one third times compared to the free acid and base, respectively. As shown from Fig. 10, as the length of terminal alkoxy chain lengths increase polarizability increases with lowering of the nematic mesophase stability. Consequently, it is predicted that the length of the alkoxy chains could affect improvements of the characteristics of the liquid crystalline materials to make them suitable for many important applications.

3.3.4. Molecular electrostatic potential (MEP). The charge distribution map (Fig. 11) for IA, IB, IC, ID was calculated using the same basis sets according to molecular electrostatic potential (MEP) negatively charged atomic sites (the red region) were mainly localized on the carboxylate moiety of the alkoxy acids, while the base part was predicted to show the least negatively charged atomic sites (blue regions). As shown from Fig. 11, the increment of alkoxy chain length does not affect either the orientation or the amount of the charge distribution map and this could illustrate the nematic mesophase of all compounds due to more end-to-end aggregation of alkoxy chains with longer chain lengths. Replacement of the linear geometry of the alkoxy acids with an angular one could permit a degree of orderliness such as the twist-bend nematic phase, especially, substitution of a symmetrical configuration by a nonsymmetrical one..$^{54,55}$

\section{Conclusions}

A new series of chair conformation supramolecular hydrogen bonded complexes were formed between the Schiff base of a phenyl nicotinate derivative and 4-alkoxybenzoic acids. All complexes exhibited a nematic phase with suitable mesophase ranges of stability. DSC and DFT calculation results revealed that as the length of the alkoxy chain increases the predicted total thermal energy and the polarizability also increases with a decrease in the nematic mesophase stability. It may be concluded that the development of these new soft materials, with different conformers with flexible terminals of a certain molecular geometry, may attract the attention of researchers for promising phase transition phenomena.

\section{Conflicts of interest}

There are no conflicts to declare.

\section{Acknowledgements}

Authors gratefully acknowledge the Deanship of Scientific Research, Taibah University for support of our research group (60333).

\section{References}

1 J.-M. Lehn, Makromol. Chem., Macromol. Symp., 1993, 69, 117.

2 T. Kato, M. Fukumasa and J. M. Frechet, Chem. Mater., 1995, 7, 368-372.

3 D. Demus, J. W. Goodby, G. W. Gray, H. W. Spiess and V. Vill, Low Molecular Weight Liquid Crystals II, Handbook of Liquid Crystals, John Wiley \& Sons, vol. 2B, 2011.

4 T. Kato, N. Hirota, A. Fujishima and J. M. Fréchet, J. Polym. Sci., Part A: Polym. Chem., 1996, 34, 57-62.

5 C. M. Paleos and D. Tsiourvas, Angew. Chem., Int. Ed. Engl., 1995, 34, 1696-1711.

6 A. J. Thote and R. B. Gupta, Ind. Eng. Chem. Res., 2003, 42, 1129-1136.

7 S. Xiao, Y. Zou, J. Wu, Y. Zhou, T. Yi, F. Li and C. Huang, J. Mater. Chem., 2007, 17, 2483-2489.

8 S. Kohmoto, Y. Someya and K. Kishikawa, Liq. Cryst., 2010, 37, 209-216.

9 M. Alaasar, C. Tschierske and M. Prehm, Liq. Cryst., 2011, 38, 925-934.

10 T. Kato, N. Mizoshita and K. Kanie, Macromol. Rapid Commun., 2001, 22, 797-814.

11 T. Kato, Science, 2002, 295, 2414-2418.

12 A. Martinez-Felipe, F. Brebner, D. Zaton, A. Concellon, S. Ahmadi, M. Piñol and L. Oriol, Molecules, 2018, 23, 2278.

13 T. Kato, J. M. J. Frechet, P. G. Wilson, T. Saito, T. Uryu, A. Fujishima, C. Jin and F. Kaneuchi, Chem. Mater., 1993, 5, 1094-1100.

14 T. Kato and J. M. Frechet, J. Am. Chem. Soc., 1989, 111, 85338534.

15 M. M. Prasad, M. M. Mohan, P. Chalapathi, A. A. Kumar and D. Potukuchi, J. Mol. Liq., 2015, 207, 294-308.

16 T. Vasanthi, P. Subhasri, R. Jayaprakasam and V. Vijayakumar, Phase Transitions, 2019, 92, 229-248.

17 P. Popov, E. K. Mann and A. Jákli, J. Mater. Chem. B, 2017, 5, 5061-5078.

18 M. H. Kishor and M. M. Mohan, J. Mol. Liq., 2019, 273, 504524. 
19 Y. Arakawa, Y. Sasaki and H. Tsuji, J. Mol. Liq., 2019, 280, 153-159.

20 M. M. Naoum, A. A. Fahmi, M. A. Alaasar and R. A. Salem, Thermochim. Acta, 2011, 517, 63-73.

21 M. M. Naoum, A. A. Fahmi, S. Z. Mohammady and A. H. Abaza, Liq. Cryst., 2010, 37, 475-486.

22 H. Ahmed, M. Hagar, M. Alaasar and M. Naoum, Liq. Cryst., 2019, 46, 550-559.

23 H. A. Ahmed, M. Hagar and A. Aljuhani, $R S C$ Adv., 2018, 8, 34937-34946.

24 H. A. Ahmed, M. Hagar, T. H. El-Sayed and R. B. Alnoman, Liq. Cryst., 2019, 1-11, DOI: 10.1080/ 02678292.2019.1566581.

25 M. Hagar, H. A. Ahmed and G. R. Saad, J. Mol. Liq., 2019, 273, 266-273.

26 M. Hagar, H. A. Ahmed and G. R. Saad, Liq. Cryst., 2018, 45, 1324-1332.

27 H. A. Ahmed and M. M. Naoum, Thermochim. Acta, 2014, 575, 122-128.

28 H. A. Ahmed, M. M. Naoum and G. R. Saad, Liq. Cryst., 2016, 43, 1259-1267.

29 H. A. Ahmed and M. M. Naoum, Liq. Cryst., 2016, 43, 222234.

30 M. Hagar, H. A. Ahmed and O. A. Alhaddad, Liq. Cryst., 2019, 1-12, DOI: 10.1080/02678292.2019.1581290.

31 M. Hagar, H. Ahmed and O. Alhaddadd, Crystals, 2018, 8, 359.

32 R. Chen, Z. An, W. Wang, X. Chen and P. Chen, Liq. Cryst., 2017, 44, 1515-1524.

33 M. Hagar, H. A. Ahmed and O. A. Alhaddadd, Crystals, 2018, 8, 359.

34 H. A. Ahmed, M. Hagar and G. Saad, Liq. Cryst., 2019, 1-11.

35 D. A. Paterson, M. Gao, Y.-K. Kim, A. Jamali, K. L. Finley, B. Robles-Hernández, S. Diez-Berart, J. Salud, M. R. de la Fuente and B. A. Timimi, Soft Matter, 2016, 12, 6827-6840.

36 D. D. Sarkar, R. Deb, N. Chakraborty, G. Mohiuddin, R. K. Nath and V. R. Nandiraju, Liq. Cryst., 2013, 40, 468-481.

37 K. Majumdar, S. Mondal and R. K. Sinha, New J. Chem., 2010, 34, 1255-1260.

38 M. Frisch, G. Trucks, H. B. Schlegel, G. Scuseria, M. Robb, J. Cheeseman, G. Scalmani, V. Barone, B. Mennucci and
G. Petersson, Gaussian 09, Revision A. 02, Gaussian, Inc., Wallingford, CT, 2009, p. 200.

39 R. Dennington, T. Keith and J. Millam, GaussView, Version 5, Semichem Inc., Shawnee Mission, 2009.

40 W. Cleland and M. M. Kreevoy, Science, 1994, 264, 18871890.

41 M. Lizu, M. Lutfor, N. Surugau, S. How and S. E. Arshad, Mol. Cryst. Liq. Cryst., 2010, 528, 64-73.

42 A. Martinez-Felipe, A. G. Cook, J. P. Abberley, R. Walker, J. M. Storey and C. T. Imrie, RSC Adv., 2016, 6, 108164108179.

43 A. Martínez-Felipe and C. T. Imrie, J. Mol. Struct., 2015, 1100, 429-437.

44 A. Ghanem and C. Noel, Mol. Cryst. Liq. Cryst., 1987, 150, 447-472.

45 D. A. Paterson, A. Martínez-Felipe, S. M. Jansze, A. TM Marcelis, J. MD Storey and C. T. Imrie, Liq. Cryst., 2015, 42, 928-939.

46 R. Walker, D. Pociecha, J. Abberley, A. Martinez-Felipe, D. Paterson, E. Forsyth, G. Lawrence, P. Henderson, J. Storey and E. Gorecka, Chem. Commun., 2018, 54, 33833386.

47 M. P. Andersson and P. Uvdal, J. Phys. Chem. A, 2005, 109, 2937-2941.

48 M. M. Naoum, A. A. Fahmi, A. A. Refaie and M. A. Alaasar, Liq. Cryst., 2012, 39, 47-61.

49 G. W. Gray, Molecular structure and the properties of liquid crystals, Academic press, 1962.

50 C. Imrie and L. Taylor, Liq. Cryst., 1989, 6, 1-10.

51 I.-C. Khoo and S.-T. Wu, Optics and nonlinear optics of liquid crystals, World Scientific, 1993.

52 D. S. Chemla, Nonlinear optical properties of organic molecules and crystals, Elsevier, 2012.

53 G. R. Meredith, J. VanDusen and D. J. Williams, Macromolecules, 1982, 15, 1385-1389.

54 C.-L. Wei, T.-C. Chen, P. Raghunath, M.-C. Lin and H.-C. Lin, RSC Adv., 2015, 5, 54629-54637.

55 S. M. Jansze, A. Martínez-Felipe, J. M. D. Storey, A. T. M. Marcelis and C. T. Imrie, Angew. Chem., Int. Ed., 2015, 54, 643-646. 